\title{
Delamination Analysis of Metal-Ceramic Multilayer Coatings Subject to Nanoindentation
}

\author{
Ryan D. Jamison ${ }^{1,2}$ and Yu-Lin Shen ${ }^{1 *}$ \\ ${ }^{1}$ Department of Mechanical Engineering \\ University of New Mexico \\ Albuquerque, New Mexico 87131 \\ USA \\ ${ }^{2}$ Component Science \& Mechanics \\ Sandia National Laboratories \\ Albuquerque, New Mexico 87185-0346 \\ USA
}

\begin{abstract}
Internal damage has been experimentally observed in aluminum $(\mathrm{Al}) /$ silicon carbide $(\mathrm{SiC})$ multilayer coatings subject to nanoindentation loading. Post-indentation characterization has identified that delamination at the coating/substrate interface is the most prominent form of damage. In this study the finite element method is employed to study the effect of delamination on indentation-derived hardness and Young's modulus. The model features alternating $\mathrm{Al} / \mathrm{SiC}$ nanolayers above a silicon $(\mathrm{Si})$ substrate, in consistence with the actual material system used in earlier experiments. Cohesive elements with a traction-separation relationship are used to facilitate delamination along the coating/substrate interface. Delamination is observed numerically to be sensitive to the critical normal and shear stresses that define the cohesive traction-separation behavior. Axial tensile stress below the edge of indentation contact is found to be the largest contributor to damage initiation and evolution. Delamination results in a decrease in both indentation-derived hardness and Young's modulus. A unique finding is that delamination can occur during the unloading process of indentation, depending on the loading condition and critical tractions.
\end{abstract}

\footnotetext{
*Email: shenyl@unm.edu
} 


\section{Introduction}

Coatings involving hybrid materials can offer intriguing properties or functionalities. One form of hybrid materials commonly encountered in natural and engineered structures is multilayers. With a total thickness of up to tens of microns and individual layer thicknesses of a few to hundreds of nanometers, these coatings see a wide range of applications including ultrahigh-strength materials, optical devices, high-performance capacitors, thermo-electric materials, high wear resistance and low friction coatings for gears, bearings, cutting tools, and thermal protective layers in aircraft and automobile engines [1-8]. In particular, thin films consisting of alternating layers of metal and ceramic can offer higher strength-to-weight ratios, less friction and wear, higher operation temperatures, corrosion resistance, and fracture toughness compared to homogenous metallic or ceramic coatings. Therefore, designing and manufacturing metal-ceramic multilayers at the micro- and nano-scales is an attractive strategy for developing a new generation of protective and infrastructure coatings, and thus have been subjects of intensive research [9-15].

Mechanical characterization of thin film and coating materials relies primarily on the nanoindentation technique. However, the scale and complexity of multilayer coatings often makes it difficult to interpret nanoindentation data. This is due to the high degree of heterogeneity pertaining to the soft/hard arrangement, along with the large interface areas. The deformation field will differ from that of a homogeneous material on which the indentation theory is based. Further, internal damage may also be induced by the indentation loading itself [16-22]. An example is shown in Fig. 1, which is a cross-section transmission electron microscopy (TEM) image of multilayer aluminum (Al) and silicon carbide (SiC) films above a silicon ( $\mathrm{Si}$ ) substrate, directly below the indentation site after nanoindentation testing [17]. The 
individual $\mathrm{Al}$ and $\mathrm{SiC}$ layers were nominally $50 \mathrm{~nm}$ thick, and loading was carried out by a Berkovich indenter to a maximum indentation depth of $1,000 \mathrm{~nm}$. It can be seen that a symmetric pattern of damage exists. Two mid-level cracks appeared below the edge of the indentation. Further below, prominent delamination of the multilayers/substrate interface has also occurred. It is worth mentioning that, aside from serving as a model system to study metal-ceramic multilayers, the $\mathrm{Al} / \mathrm{SiC}$ multilayer system is also being considered as reflective coatings in ultraviolet applications [23,24].

One immediate question is how the internal damage would influence the indentationmeasured properties, such as hardness and elastic modulus. It is also unclear at what stage of the nanoindentation testing did cracking occur. It is virtually impossible to answer these questions by common experimental observations. In the same material system, any experimentally measured quantities will have been affected by the damage already (without a "clean" set of results to compare with). Therefore in this study we employ numerical finite element modeling to study the evolution of delamination and its effect on indentation response. Cohesive zone elements were built into the model at the coating/substrate interface, to allow for crack development during indentation loading. Comparisons of results with and without the cohesive zone will then provide quantitative insight to the effect of cracking. The effects of cohesive zone model parameters were also investigated.

\section{Model Description}

The finite element model consists of 41 alternating $\mathrm{Al}$ and $\mathrm{SiC}$ thin films on a substrate of $\mathrm{Si}$. This geometry corresponds to the actual multilayer system shown in Fig. 1 studied previously [16,17]. A schematic of the model is shown in Fig. 2. Both the top layer (to be in contact with the indenter) and the bottom layer (adjacent to the Si substrate) are Al. A conical diamond indenter 
with a semi-angle of $70.3^{\circ}$ is assumed. This indenter geometry results in the same projected contact area, for a given depth, as that of a Berkovich indenter in common nanoindentation experiments. Use of the conical indenter is a practical way to model the indentation process in a two-dimensional setting [25]. The model is axisymmetric, with the left boundary being the symmetry axis. The overall size of the entire specimen is $40 \mu \mathrm{m}$ in lateral span (radius) and 43 $\mu \mathrm{m}$ in height. The thicknesses of the individual $\mathrm{Al}$ and $\mathrm{SiC}$ layers are $50 \mathrm{~nm}$ each. In addition to the multilayer model, a separate reference model is also used. The reference model consists of a $1 \mu \mathrm{m}$-thick single-layer $\mathrm{Al}$ film on the same $\mathrm{Si}$ substrate. The reference model is used to determine if the indentation model is sensitive to cohesive elements at the Si interface.

During deformation the left and bottom boundaries are allowed to displace only in the axial and radial directions, respectively. The right boundary is not constrained. The top Al surface, when not in contact with the indenter, is also free to move. When contact with the indenter is established, the surface portion engaged by the indenter directly interacts with the indenter. The coefficient of friction between the indenter and the top surface is 0.1 , which is a typical value for the diamond/metal contact surface.

Approximately 100,000 fully integrated linear axisymmetric elements are used in the finite element model with a finer mesh size near the upper-left corner of the test material. The element size near the indenter tip is approximately $8 \mathrm{~nm}$ and increases in size far from the indenter. The indenter consists of approximately 8,000 elements with an element size of approximately $15 \mathrm{~nm}$ near the tip. The mesh was created using CUBIT (Sandia National Laboratories; Albuquerque, NM, U.S.A.). The finite element program ABAQUS (Version 6.13, Dassault Systemes Simulia Corp.; Providence, RI, U.S.A.) was used to carry out the analysis.

\subsection{Material parameters}


The Young's modulus for $\mathrm{Al}$ is assumed to be $59 \mathrm{GPa}$. Nanoindentation measurement of single-layer $\mathrm{Al}$ provided the modulus for this material. The Poisson's ratio for $\mathrm{Al}$ is 0.33 . The plastic response of $\mathrm{Al}$ was based on the tensile loading data of single-layer $\mathrm{Al}$ with initial yield strength of $200 \mathrm{MPa}$. Rate-independent isotropic elastic-plastic response was assumed, with plastic yielding following the von Mises criterion with isotropic hardening and the incremental flow theory. The piecewise linear strain hardening response features hardening slopes of 199.33 MPa from initial yielding up to the strain of $50.51 \%$ and then $39.97 \mathrm{MPa}$ up to the strain of 300.68\%, beyond which perfect plasticity ensues.

The Young's modulus for $\mathrm{SiC}$ is assumed to be $277 \mathrm{GPa}$. Nanoindentation measurement of single-layer $\mathrm{SiC}$ films provided the modulus. The somewhat lower modulus of $\mathrm{SiC}$ relative to crystalline $\mathrm{SiC}$ is due to the fact that the physical vapor deposited $\mathrm{SiC}$ layers in the present case were amorphous [26]. The Poisson's ratio for $\mathrm{SiC}$ is taken as 0.17 . $\mathrm{SiC}$ is a very brittle material; nevertheless, a very high yield point of $8,770 \mathrm{MPa}$ (estimated from the indentation hardness of a single-layer $\mathrm{SiC}$ film) was used followed by perfect plasticity. The plasticity assumption is necessitated by the fact that a purely elastic $\mathrm{SiC}$ in the model will generate unrealistically high loads during the indentation simulation, and it is validated by the fact that in the experiment the $\mathrm{SiC}$ layers exhibited a glassy/plastic-type response due to the amorphous nature of the film.

Both the Si substrate and diamond indenter are assumed to remain elastic. The Young's modulus and Poisson's ratio of the Si substrate are $187 \mathrm{GPa}$ and 0.28 , respectively. The Young's modulus and Poisson's ratio of the diamond indenter are 1,141 GPa and 0.07, respectively. All the interfaces between different materials in the composite structure are modeled as perfectly bonded, unless otherwise noted in Section 2.2 (the cohesive zone allowing for delamination). The same set of material parameters were used previously to numerically study basic 
nanoindentation response [16], cyclic indentation behavior [27], the effect of layer undulation [28] and the effect of unloading induced plasticity [29] in the Al/SiC multilayer coatings. In the present work the effect of delamination is investigated.

The indentation-derived elastic modulus and hardness were directly obtained from the finite element modeling. The contact stiffness at the onset of unloading, $S$, can be calculated using Eqs. (1) and (2) [30]

$$
S=\beta \frac{2}{\sqrt{\pi}} E_{e f f} \sqrt{A}
$$

with

$$
\frac{1}{E_{e f f}}=\frac{1-v^{2}}{E}+\frac{1-v_{i}^{2}}{E_{i}}
$$

where $A$ is the projected contact area at onset of unloading, $\beta$ is the indenter geometry-dependent dimensionless parameter, $E$ and $v$ are the Young's modulus and Poisson's ratio, respectively, of the material being indented (composite layers), and $E_{\mathrm{i}}$ and $v_{\mathrm{i}}$ are the Young's modulus and Poisson's ratio, respectively, of the diamond indenter. In the simulation, the parameter $\beta$ was first calibrated with a pure $\mathrm{Al}$ body of the same geometry as the entire multilayers/substrate assembly, and a value of 1.06 was determined. This was accomplished by constraining the indentation-derived Young's modulus to be equivalent to the input value used in the finite element analysis. When calculating the projected contact area $A$, the last nodal point on the top surface in contact with the indenter was identified in the deformed mesh. Thus, the effect of pileup was automatically taken into consideration. The determination of the composite modulus $E$ requires a known Poisson's ratio, $v$. Here a separate finite element analysis of uniaxial loading 
of the $\mathrm{Al} / \mathrm{SiC}$ laminates was used to determine $v$ [31], which results in a value of 0.25 . Finally, the hardness of the material, $H$, is given by

$$
H=\frac{P}{A}
$$

with $P$ being the load at a given indentation depth and $A$ the corresponding projected contact area.

2.2 Modeling delamination

Postmortem analysis has shown that indentation-induced damage can appear in the mid-layer region and at the interface between multilayer coating and substrate, generally underneath the edge of the indentation contact (Fig. 1). Due to the fact that cracking along the multilayer/substrate interface is more extensive, and that the mid-layer cracks were observed to be within the metal or ceramic layers [16,17], in this study cohesive zone elements are only used to model delamination between the bottommost Al layer and Si substrate.

The most common method used to describe the constitutive response of cohesive elements is with a traction-separation law (Fig. 3). This relationship is useful for modeling delamination because the user can relate stresses to separation at the interface in both the normal and tangential directions. The traction-separation law is composed of two main components, loading and unloading. The relationship has a stiffness of $K$ until a critical stress $\left(t^{0}\right)$, or interface strength, at displacement $\delta$ is reached. Upon reaching the critical stress, damage occurs for $\delta>$ $\delta_{0}$. Damage continues until a separation distance of $\delta_{\text {sep }}$ is reached, at which point the fracture energy has been released. At the point of failure, the traction-separation relationship accounts for an amount of energy equal to the critical fracture energy of the interface. Although the 
constitutive response has a physical basis, it is nonetheless a phenomenological representation of a complex phenomenon.

Damage initiation is characterized by the maximum nominal stress ratio, defined as

$$
\max \left\{\frac{\left\langle t_{n}\right\rangle}{t_{n}^{0}}, \frac{t_{s}}{t_{s}^{0}}\right\}=1
$$

where $t_{n}$ is the normal traction modified with the Macaulay bracket with the standard interpretation (i.e. damage does not initiate in a pure compressive state), $t_{n}^{0}$ is the critical tensile stress, $t_{s}$ is the shear stress, and $t_{s}^{0}$ is the critical shear stress. When the maximum of these ratios reach 1.0, damage initiates and the cohesive element begins to accumulate damage.

Once damage initiates, damage evolution is assumed to be linear and based on effective displacement (separation). Damage evolution is controlled by a scalar damage variable, $D$, which represents the overall damage in the cohesive element. The variable $D$ monotonically evolves from 0 to 1 upon further loading post damage initiation. As damage evolution is based on separation, it is useful to define an effective displacement as

$$
\delta_{m}=\sqrt{\left\langle\delta_{n}\right\rangle^{2}+\delta_{s}^{2}}
$$

where $\delta_{n}$ is the tensile-only component of the normal separation and $\delta_{s}$ is the shear separation. The scalar damage variable is then defined as

$$
D=\frac{\delta_{m}^{f}\left(\delta_{m}^{\max }-\delta_{m}^{0}\right)}{\delta_{m}^{\max }\left(\delta_{m}^{f}-\delta_{m}^{0}\right)}
$$

where $\delta_{m}^{f}$ is the effective displacement at complete failure, $\delta_{m}^{0}$ is the effective displacement at damage initiation, and $\delta_{m}^{\max }$ is the maximum value of the effective displacement attained during the loading history. Once $D$ attains a value of unity, the damage evolution is complete and the 
element is deleted. The combination of damage initiation and evolution completely define the constitutive behavior of the cohesive element.

The interface fracture energy for a given material system is very sensitive to processing, detailed interface chemistry, testing environment, and the relative magnitude of shear traction to normal traction acting on the interface ahead of the delamination [32]. The assignment of damage parameters is therefore subject to uncertainty. In the present modeling the nominal values of both $t_{n}^{0}$ and $t_{s}^{0}$ are assumed to be $200 \mathrm{MPa}$. They are also varied between 200 and 400 MPa to gain further insight into how the interface behaves. The critical separation, $\delta_{m}^{f}$, is assumed to be $0.4 \mathrm{~nm}$. During indentation loading very large compressive stresses are generated below the indenter. However, vertical tensile stresses do exist in a region below and outside of the contact edge (see results presented Section 3.2). The choice of delamination initiation stress corroborates with the simulated tensile stress range where interfacial cracking was observed experimentally. The elastic properties of the cohesive element are based on the bulk Al properties. The elastic moduli in the normal and shear directions are 59 and $28 \mathrm{GPa}$, respectively. In the finite element model, the cohesive zone is a separate element block that is placed at a material interface. Since the physical thickness of the material interface is nearly zero, the cohesive element is assumed to have zero initial thickness. In both the multilayer model and the single-Al reference model, cohesive elements are inserted at the Al/Si substrate interface.

\section{Results and Discussion}

The reference case of a single-layer Al is considered first, in Section 3.1. Results from the perfectly bonded multilayers (no cohesive elements) are presented in Section 3.2. The purpose is to establish basic tensile and shear stress patterns under indentation loading, which are helpful in 
subsequent discussions. Section 3.3 is then devoted to the delamination analysis of multilayers incorporating the cohesive zone at the multilayer coating/substrate interface.

\subsection{Single-layer Al film}

The single-layer model is a useful tool to determine if the presence of the cohesive elements have any effect on the load-displacement response. The $\mathrm{Al}$ is $1 \mu \mathrm{m}$ thick and is joined to a thick Si substrate with cohesive elements. The indentation load-displacement response of the singlelayer $\mathrm{Al}$ film with and without cohesive elements is shown in Fig.4 for a maximum indentation depth of $500 \mathrm{~nm}$. It can be seen that the load-displacement response of both models are identical. This suggests that the use of cohesive elements at the interface does not affect the indentation modeling results. Additionally, from examination of the deformed structure, the single-layer film is not susceptible to delamination at the $\mathrm{Al} / \mathrm{Si}$ interface.

The indentation-derived hardness and Young's modulus for the single-layer film is shown in Fig. 5 for an indentation range of 10 to $50 \%$ of film thickness. In each image, the results of the model with cohesive elements are very similar to the model without. Although they are similar, they are not identical. The small difference in both the hardness and modulus can be attributed to the compliance at the film/substrate interface, which includes both local sliding and Mode I "opening" that is below the critical traction. The cohesive elements allow for the interface to shear. The shear at the interface leads to a small change in the projected contact area; hence, the small difference in the models. During both loading and unloading, the cohesive elements remain elastic, as damage is never initiated. The presence of cohesive elements at the film/substrate interface in a single-layer $\mathrm{Al}$ film was shown to have little effect on indentation-derived variables. 


\subsection{Perfectly bonded multilayers}

The $\mathrm{Al} / \mathrm{SiC}$ multilayer structure results in a much more complex indentation response. As a start, representative stress fields are first shown for the perfectly bonded multilayer coating on a Si substrate, without any cohesive zone included in the model. Figure 6 shows the contour plots of axial stress along the indentation direction, $\sigma_{22}$, when the indentation depth reaches (a) 600 $\mathrm{nm}$ and (b) after complete unloading. Directly under the indentation contact a very large compressive stress develops. However, in Fig. 6 the contour levels were adjusted so as to highlight the tensile $\sigma_{22}$ stresses evolved during loading and unloading (otherwise the localized tensile stresses would be largely "hidden" by the predominant compressive field). It can be seen that, under indentation, tensile stresses in the vertical direction have developed in certain areas below and slightly outside the edge of indentation. The stress magnitude can be significant, well over $200 \mathrm{MPa}$ in many $\mathrm{Al}$ and $\mathrm{SiC}$ layers as well as in a small region of the Si substrate (Fig. 6(a)). It is notable that the delamination observed in Fig. 1 falls into the region where large tensile $\sigma_{22}$ stresses are seen in the modeling.

Upon unloading, Fig. 6(b), the region with high tensile stresses has shifted slightly inward. It is noticed that the area with tensile $\sigma_{22}$ stresses has actually expanded during unloading. The enhancement of tensile stress is due to the fact that the heavily compressed material directly below the indentation contact has undergone elastic recovery during unloading. This imposes a vertical pulling action on the region already under tension. The evolution of axial stress suggests the possibility that internal cracking in the structure can be induced during the unloading phase of the indentation. This will be illustrated by the damage modeling presented in Section 3.3 below. 
Figures 7(a) and (b) show the contour plots of shear stress $\sigma_{12}$ near the indentation contact, at an indentation depth of $600 \mathrm{~nm}$ and after complete unloading, respectively. In Fig. 7(a) a highly stressed region within the Si substrate and at the coating interface is evident. Shear traction induced delamination is therefore possible, depending on the relative normal and shear strength of the interface. After unloading, the magnitude of shear stress is significantly relaxed, particularly at the multilayer/substrate interface, Fig. 7(b).

\subsection{Multilayers with cohesive zone elements}

When the cohesive zone elements are built into the interface between the multilayer coating and substrate, delamination then becomes a possibility. The indentation load-displacement response, hardness, Young's modulus, and delamination evolution are now presented.

\subsubsection{Load-displacement response}

Figure 8 shows the load-displacement response during indentation loading and unloading with various maximum indentation depths. In this figure the critical normal $\left(t_{n}^{0}\right)$ and shear $\left(t_{s}^{0}\right)$ stresses are varied between 200 and $400 \mathrm{MPa}$, and the circles denote the load-depth points at which delamination was observed to occur in the model. As discussed in greater detail below, in Figs. 8(a) to (c), delamination occurs at one loading and unloading event, and in Fig. 8(d), delamination occurs once during loading and twice during unloading.

In Figs. 8(a) and (b), the normal critical traction is held constant at $200 \mathrm{MPa}$. In both images, delamination occurs at $23 \mathrm{mN}$ during loading and at $12 \mathrm{mN}$ during unloading. The increase in the shear critical traction, Fig. 8(b), does not cause a change in the load at which delamination occurs. This suggests that delamination in these two sets of models is dominated by the tensile axial (normal) stress at the interface. In Fig. 8(c), the load at which delamination occurs is 
increased. During loading $(56 \mathrm{mN})$, damage initiation is caused by shear at the interface. During unloading $(34 \mathrm{mN})$, damage initiation is dominated by a tensile axial stress at the interface. In Fig. 8(d), delamination occurs at three separate loading conditions. First, delamination occurs during loading at $62 \mathrm{mN}$ and damage initiation is driven by both axial and shear stresses. For both unloading-induced delamination events ( 34 and $61 \mathrm{mN}$ ) tensile axial stress is the main factor in damage initiation. From this set of data, it is observed that the delamination during loading can be attributed to both tensile axial and shear stresses, while delamination during unloading is dominated by tensile axial stress.

One case of the load-displacement response, with and without the cohesive elements, is shown in Fig. 9 with the maximum indentation depth of $600 \mathrm{~nm}$. The critical tractions in both the normal and shear directions are assumed to be $200 \mathrm{MPa}$. It can be seen that the indentation loading and unloading response is very similar for the perfect "bonded" case and the one with the cohesive zone. The peak loads differ by less than $2 \%$, even though delamination has occurred in the "cohesive" case (see also Fig. 8(a)). The observation suggests that the presence of cohesive elements do not, in themselves, cause any significant difference in the load-displacement response. While the peak load may only be slightly affected, a change in the projected contact area or unloading behavior can cause more substantial differences in derived hardness or Young's modulus, as presented below.

\subsubsection{Indentation-derived variables}

The hardness as a function of indentation depth of the multilayer $\mathrm{Al} / \mathrm{SiC}$ with and without cohesive elements is shown Fig.10, for a range of critical normal and shear tractions from 200 to $400 \mathrm{MPa}$ (as indicated by the labels " $\mathrm{n} 200-\mathrm{s} 200$ " etc.). Up to an indentation depth of $600 \mathrm{~nm}$, the difference in the hardness result is small. Above $600 \mathrm{~nm}$, the difference becomes larger, with a 
maximum deviation in hardness reaching $-13 \%$ at the deepest indentation considered. A change in hardness comes from both a change in peak load as well as a change in the projected contact area. The cohesive elements, particularly post-delamination, allow the structure to move more freely, resulting in an increase in the projected contact area.

Over a large portion of the indentation range considered, the hardness does not vary by more than $10 \%$, even though delamination has occurred. The Young's modulus on the other hand is much more sensitive to delamination. The indentation-derived Young's modulus of the multilayer $\mathrm{Al} / \mathrm{SiC}$ coating with and without cohesive elements is shown in Fig. 11. At an indentation depth of $300 \mathrm{~nm}$, the Young's modulus varies by as much as $6 \%$ and the difference grows to $22 \%$ at $900 \mathrm{~nm}$ among the cases considered. The most significant difference in Young's modulus comes after delamination has occurred, i.e. when the slope of the modulus-depth response becomes negative.

In general, the presence of the cohesive elements results in a decrease in the derived modulus (pre-delamination). Similar to the hardness, this softening behavior can be attributed to multiple factors. First, the projected contact area increases as described above. For example, for the case of $t_{n}^{0}=400 \mathrm{MPa}$ and $t_{s}^{0}=400 \mathrm{MPa}$, the projected contact area is $11 \%$ greater than the perfectly bonded model at an indentation depth of $900 \mathrm{~nm}$. Second, the contact stiffness at the onset of unloading, $S$, decreases when the interface is modeled with cohesive elements. For the same material set (n400-s400), at an indentation depth of $900 \mathrm{~nm}, S$ has decreased by $15 \%$. The combination of these two effects causes the large decrease in the indentation-derived Young's modulus.

In the multilayers the hard $\mathrm{SiC}$ imparts a constraint on the $\mathrm{Al}$ layers. During indentation, the $\mathrm{Al}$ is forced to flow plastically away from the indentation site. In addition, SiC layers also exhibit 
significant flexibility due to their small thickness and the tight sandwich structure. Therefore there is a limited extent of pile-up at the edge of the indentation contact as observed in Fig. 6(a). Allowing for interfacial damage effectively relaxes the constraints created by the $\mathrm{Al} / \mathrm{SiC}$ multilayers. Prior to damage initiation, interfacial compliance partially relieves the constraint so plastic flow occurs more easily, effectively increasing the contact area. After damage occurs at the $\mathrm{Al} / \mathrm{Si}$ interface, the contact stiffness decreases. Damage at the interface also partially relaxes the influence of the Si substrate. As a consequence, while delamination occurs at bottom of the multilayer coating, the indentation-measured elastic modulus can still be significantly affected.

\subsubsection{Indentation-induced damage}

It was observed in the present study that, depending on the load applied and choice of parameters, damage initiation can be induced by either normal stress or shear stress. Take the case of maximum indentation depth of $600 \mathrm{~nm}$ in Fig. 11 as an example: the perfectly bonded model has a modulus $18 \%$ greater than that with "n200-s200" cohesive elements. Delamination occurs at a load of $23 \mathrm{mN}$ during loading (Fig. 8(a)). Figures 12(a) and (b) show the contour plots of axial stress $\left(\sigma_{22}\right)$ at $98 \%$ of the peak load during unloading, in the models with perfect bonding and "n200-s200" cohesive elements, respectively. Note that between $98-100 \%$ peak load is the range in which a linear regression is used to fit the load-displacement data during unloading to calculate the contact stiffness $S$. At $98 \%$ of peak load, the axial stress is much lower in the cohesive element model than the perfectly bonded model. Delamination allows the multilayer film to move away from the substrate more freely during unloading; therefore, the contact stiffness at the onset of unloading is decreased. Note that delamination in Fig. 12(b) appears at the same general location as observed in experiment, Fig. 1. 
The axial and shear stresses in the multilayer structure post-unloading are now considered. In this presentation the critical normal and shear stresses of the cohesive elements are still equal to $200 \mathrm{MPa}$. Figures 13(a) and (b) show the contour plots of axial stress $\sigma_{22}$ and shear stress $\sigma_{12}$, respectively, after complete unloading from the maximum indentation depth of $600 \mathrm{~nm}$. A comparison between Fig. 13(a) with Fig. 6(b) can be made, with Fig. 6(b) pertaining to a perfectly bonded coating. In Fig. 6(b) the large axial tensile stress extends through nearly every layer and into the substrate. But in Fig. 13(a) the volume of the multilayers subject to these high tensile stresses has decreased significantly. The large opening at the multilayer/substrate interface observed in Fig. 13(a) is promoted by the unloading action. When comparing Fig. 13(b) with Fig. 7(b) (where a perfectly bonded model is used), it can be observed that the presence of delamination does not have a significant effect on the magnitude and distribution of shear stress. A discussed in Section 3.3.1, damage initiation and evolution in this case is dominated by the axial tensile stress at the multilayer/substrate interface.

Fig. 8 shows that for a specific combination of critical tensile and shear tractions, delamination can occur at the multilayer/substrate interface during the unloading process. An example is shown in Fig. 14, with contour plots of the axial stress $\sigma_{22}$ (a) at the peak load and (b) during unloading. In this figure, the normal and shear critical tractions are both assumed to be $200 \mathrm{MPa}$ and the maximum indentation depth is $400 \mathrm{~nm}$. When the specimen is fully loaded, Fig. 14(a), there is a large tensile stress across the multilayer/substrate interface. After unloading begins, Fig. 14(b), the tensile axial stress results in damage initiation and crack growth. This can be observed by the separation at the interface and the stress contour at the crack front. At the load shown in Fig. 14(b), $10.9 \mathrm{mN}$, tensile stress is not completely relieved at the interface and 
further damage can occur. This illustrates that damage initiation can occur not only during loading but also during the unloading process.

As described in Section 2.2, the present study only considered delamination at the interface between the bottom $\mathrm{Al}$ layer and Si substrate. If the cohesive elements were also applied to the individual $\mathrm{Al} / \mathrm{SiC}$ interfaces, then the smaller mid-level cracks observed in Fig. 1 could potentially be simulated. The outcome may depend on the choice of damage parameters which are largely unknown. Since electron microcopy observations suggested that the mid-level cracks appear within the layers and not along the interface, they were not included in the current analysis. Further experimental and numerical characterizations of these damage occurrences are recommended.

\section{Conclusions}

This study is devoted to nanoindentation-induced delamination along the interface between the $\mathrm{Al} / \mathrm{SiC}$ multilayer coating and $\mathrm{Si}$ substrate, using finite element analyses. Cohesive elements with a traction-separation relationship were used to facilitate delamination. As a reference case, the single-layer Al film did not exhibit any delamination and the presence of cohesive elements did not adversely affect the indentation-derived variables. Delamination was observed in the $\mathrm{Al} / \mathrm{SiC}$ multilayer model, underneath the indentation contact edge and spanning outward, as was observed experimentally [17]. Delamination was found to be sensitive to the critical normal and shear stresses that define the cohesive traction-separation behavior. The load-displacement response of the multilayer film is not significantly different between perfectly bonded and cohesive material interfaces. This suggests that it may be difficult to experimentally observe delamination solely from load-displacement data. Axial tensile stress below the indentation contact edge was found to be the largest contributor to damage initiation and evolution. Within 
the indentation depth range studied, interfacial damage resulted in a decrease in both indentationderived hardness and Young's modulus, by as much as 13 and 22\%, respectively. Furthermore, a unique finding is that delamination may occur during the unloading process of indentation, depending on the loading condition and critical tractions.

\section{Acknowledgements}

Sandia National Laboratories is a multiprogram laboratory managed and operated by Sandia Corporation, a wholly owned subsidiary of Lockheed Martin Corporation, for the U.S.

Department of Energy's National Nuclear Security Administration under contract DE- AC0494AL85000. 


\section{References}

[1] S.W. Ko, T. Dechakupt, C.A. Randall, S. Trolier-McKinstry, M. Randall, A. Tajuddin, J. Electroceram. 24 (2010) 161-169.

[2] T. Koseki, J. Inoue, S. Nambu, Mater. Trans. 55 (2014) 227-237.

[3] Y. Sahin, Surf. Coat. Technol. 199 (2005) 112-117.

[4] L. Ghalandari, M.M. Moshksar, J. Alloys Compd. 506 (2010) 172-178.

[5] A.A. Voevodin, J.M. Schneider, C. Rebholz, A. Matthews, Tribol. Int. 29 (1996) 559-570.

[6] M.P. Schmitt, A.K. Rai, R. Bhattacharya, D.M. Zhu, D.E. Wolfe, Surf. Coat. Technol. 251 (2014) 56-63.

[7] D.L. Windt, J.A. Bellotti, Appl. Opt. 48 (2009) 4932-4941.

[8] A. Ziani, F. Delmotte, C. Le Paven-Thivet, E. Meltchakov, A. Jerome, M. Roulliay, F. Bridou, K. Gasc, Thin Solid Films 552 (2014) 62-67.

[9] I. Knorr, N.M. Cordero, E.T. Lilleodden, C.A. Volkert, Acta Mater. 61 (2013) 4984-4995.

[10] D. Bhattacharyya, N.A. Mara, P. Dickerson, R.G. Hoagland, A. Misra, Acta Mater. 59 (2011) 3804-3816.

[11] D.R.P. Singh, N. Chawla, G. Tang, Y.-L. Shen, Acta Mater. 58 (2010) 6628-6636.

[12] J. Romero, A. Lousa, E. Martinez, J. Esteve, Surf. Coat. Technol. 163-164 (2003) 392-397.

[13] M.A. Phillips, B.M. Clemens, W.D. Nix, Acta Mater. 51 (2003) 3171-3184.

[14] Y.-C. Wang, A. Misra, R.G. Hoagland, Scripta Mater. 54 (2006) 1593-1598. 
[15] A.S. Budiman, S.-M. Han, N. Li, Q-M. Wei, P. Dickerson, N. Tamura, M. Kunz, A. Misra, J. Mater. Res. 27 (2012) 599-611.

[16] G. Tang, Y.-L. Shen, D.R.P. Singh, N. Chawla, Acta Mater. 58 (2010) 2033-2044.

[17] P.L. Sun, J.P. Chu, T.Y. Lin, Y.-L. Shen, N. Chawla, Mater. Sci. Engng. A 257 (2010) 2985-2992.

[18] M. Parlinska-Wojtan, S. Meier, J. Patscheider, Thin Solid Films 518 (2010) 4890-4897.

[19] D. Wang, T. Kups, J. Schawohl, P. Schaaf, J. Mater. Sci. - Mater. Electron. 23 (2012) 1077-1082.

[20] P. Wiecinski, J. Smolik, H. Garbacz, K.J. Kurzydlowski, Surf. Coat. Technol. 240 (2014) $23-31$.

[21] J.J. Chen, S.J. Bull, Thin Solid Films 517 (2009) 3704-3711.

[22] W.M. Mook, R. Raghavan, J.K. Baldwin, D. Frey, J. Michler, N.A. Mara, A. Misra, Mater. Res. Lett. 1 (2013) 102-108.

[23] D.L. Windt, J.A. Bellotti, Appl. Opt. 48 (2009) 4932-4941.

[24] A. Ziani, F. Delmotte, C. Le Paven-Thivet, E. Meltchakov, A. Jerome, M. Roulliay, F. Bridou, K. Gasc, Thin Solid Films 552 (2014) 62-67.

[25] A.C. Fischer-Cripps, Nanoindentation, Springer, New York, 2002.

[26] X. Deng, N. Chawla, K.K. Chawla, M. Koopman, J.P. Chu, Adv. Engng. Mater. 7 (2005) 1099-1108. 
[27] Y.-L. Shen, C.B. Blada, J.J. Williams, N. Chawla, Mater. Sci. Engng. A 557 (2012) 119125.

[28] R.D. Jamison, Y.-L. Shen, J. Composite Mater. DOI:10.1177/0021998315576768.

[29] R.D. Jamison, Y.-L. Shen, J. Mater. Res. 30 (2015) 2279-2290.

[30] W.C. Oliver, G.M. Pharr, J. Mater. Res. 7 (1992) 1564-1583.

[31] Y.-L. Shen, Constrained Deformation of Materials, Springer, New York, 2010.

[32] L.B. Freund, S. Suresh, Thin Film Materials, Cambridge University Press, Cambridge, 2003. 


\section{Figure Captions}

Fig. 1 TEM image of post-indented Al/SiC multilayers, revealing indentation-induced internal cracks and permanent deformation [17].

Fig. 2 Schematic showing the $\mathrm{Al} / \mathrm{SiC}$ multilayers above a Si substrate and the boundary conditions used in the axisymmetric model.

Fig. 3 A graphical representation of the traction-separation relationship that defines the cohesive element response.

Fig. 4 Simulated load-displacement response, during indentation loading and unloading, for a single-layer Al film with and without cohesive elements. "Bonded" refers to a perfect interface (no cohesive elements) and "Cohesive" refers to an interface composed of cohesive elements.

Fig. 5 Indentation-derived (a) hardness and (b) Young's modulus of a single-layer Al film with and without cohesive elements. "Bonded" refers to a perfect interface (no cohesive elements) and "Cohesive" refers to an interface composed of cohesive elements.

Fig. 6 Contour plots of axial stress $\sigma_{22}$ (along the indentation direction) in the perfectly bonded multilayer $\mathrm{Al} / \mathrm{SiC}$ model, when (a) the indentation reaches its peak depth at $600 \mathrm{~nm}$, and (b) after complete unloading.

Fig. 7 Contour plots of shear stress $\sigma_{12}$ in the perfectly bonded multilayer $\mathrm{Al} / \mathrm{SiC}$ model, when (a) the indentation reaches its peak depth at $600 \mathrm{~nm}$, and (b) after complete unloading.

Fig. 8 The indentation load-displacement response of the multilayer Al/SiC coating with cohesive elements at the $\mathrm{Al} / \mathrm{Si}$ interface with variations in normal and shear critical tractions. (a) $t_{n}^{0}=200 \mathrm{MPa}, t_{s}^{0}=200 \mathrm{MPa}$ (b) $t_{n}^{0}=200 \mathrm{MPa}, t_{s}^{0}=400 \mathrm{MPa}$ (c) $t_{n}^{0}=400$ $\mathrm{MPa}, t_{s}^{0}=200 \mathrm{MPa}(\mathrm{d}) t_{n}^{0}=400 \mathrm{MPa}, t_{s}^{0}=400 \mathrm{MPa}$. Circles denote the load-depth instances at which delamination occurs.

Fig. 9 Indentation load-displacement response of the multilayer $\mathrm{Al} / \mathrm{SiC}$ coating with cohesive elements at the $\mathrm{Al} / \mathrm{Si}$ interface, with a maximum indentation depth of $600 \mathrm{~nm}$.

Fig. 10 Indentation-derived hardness of the multilayer $\mathrm{Al} / \mathrm{SiC}$ coating for a range of critical normal and shear tractions. The labels "n200-s200", "n200-s400", "n400-s200", and "n400-s400" represent the critical normal (n) and shear (s) tractions at the coating/substrate interface. The perfectly bonded model is the solid line.

Fig. 11 Indentation-derived Young's modulus of the multilayer $\mathrm{Al} / \mathrm{SiC}$ coating for a range of critical normal and shear tractions. The labels "n200-s200", "n200-s400", "n400-s200", and "n400-s400" represent the critical normal (n) and shear (s) tractions at the coating/substrate interface. The perfectly bonded model is the solid line. 
Fig. 12 Contour plots of axial stress $\left(\sigma_{22}\right)$ at $98 \%$ of the peak load during unloading, for a peak indentation depth of $600 \mathrm{~nm}$ in the models with (a) perfect bonding and (b) cohesive elements at the coating/substrate interface.

Fig. 13 Contour plots of (a) axial stress $\sigma_{22}$ and (b) shear stress $\sigma_{12}$ in the multilayer $\mathrm{Al} / \mathrm{SiC}$ structure after complete unloading from the maximum indentation depth of $600 \mathrm{~nm}$. Unloading is seen to enhance the opening at the coating/substrate interface.

Fig. 14 Contour plots of axial stress $\sigma_{22}$ in the multilayer $\mathrm{Al} / \mathrm{SiC}$ structure (a) at the peak indentation depth of $400 \mathrm{~nm}$ and (b) after unloading to $10.9 \mathrm{mN}$. Delamination in the case is completely generated during unloading. 


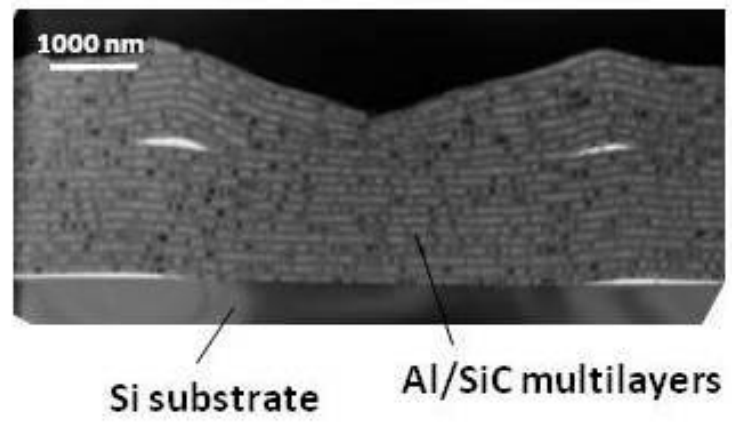

Fig. 1 TEM image of post-indented $\mathrm{Al} / \mathrm{SiC}$ multilayers, revealing indentation-induced internal cracks and permanent deformation [17]. 


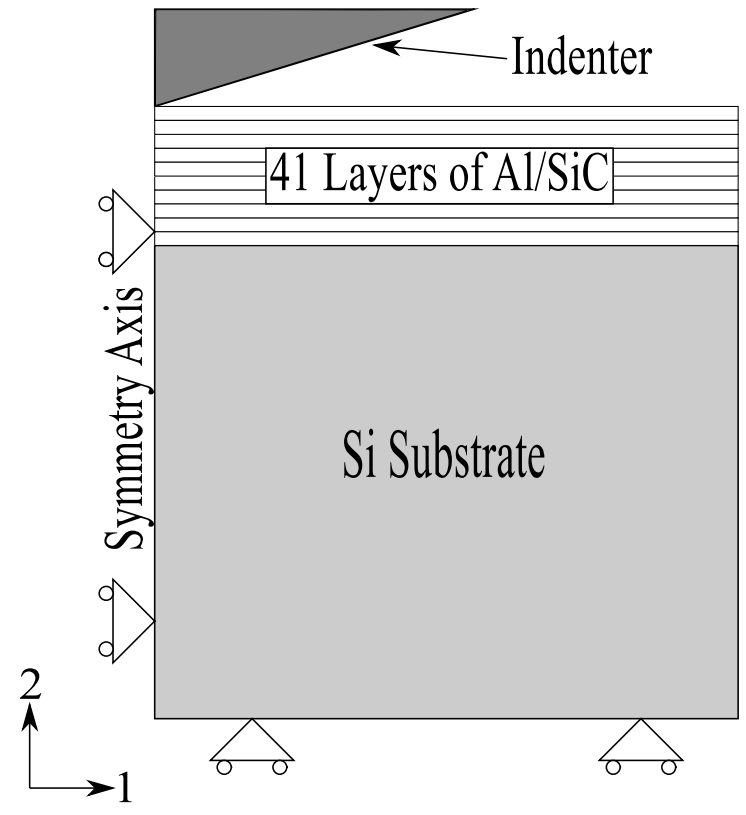

Fig. 2 Schematic showing the $\mathrm{Al} / \mathrm{SiC}$ multilayers above a Si substrate and the boundary conditions used in the axisymmetric model. 


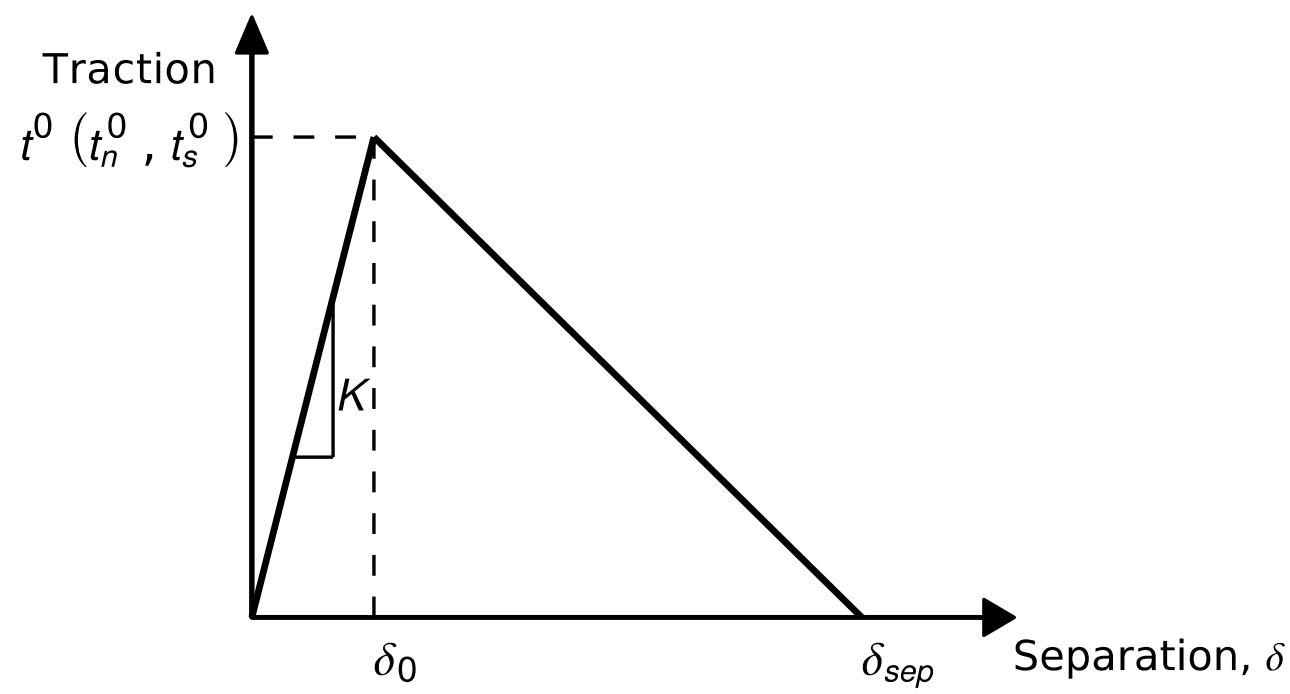

Fig. 3 A graphical representation of the traction-separation relationship that defines the cohesive element response. 


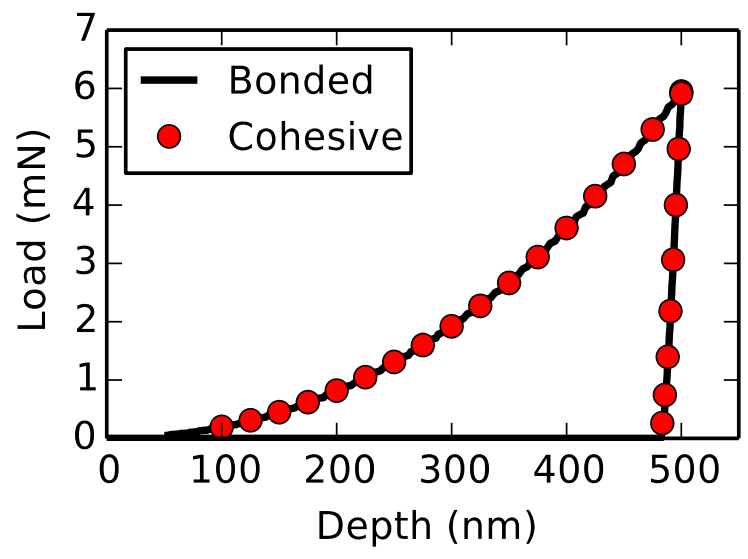

Fig. 4 Simulated load-displacement response, during indentation loading and unloading, for a single-layer Al film with and without cohesive elements. "Bonded" refers to a perfect interface (no cohesive elements) and "Cohesive" refers to an interface composed of cohesive elements. 

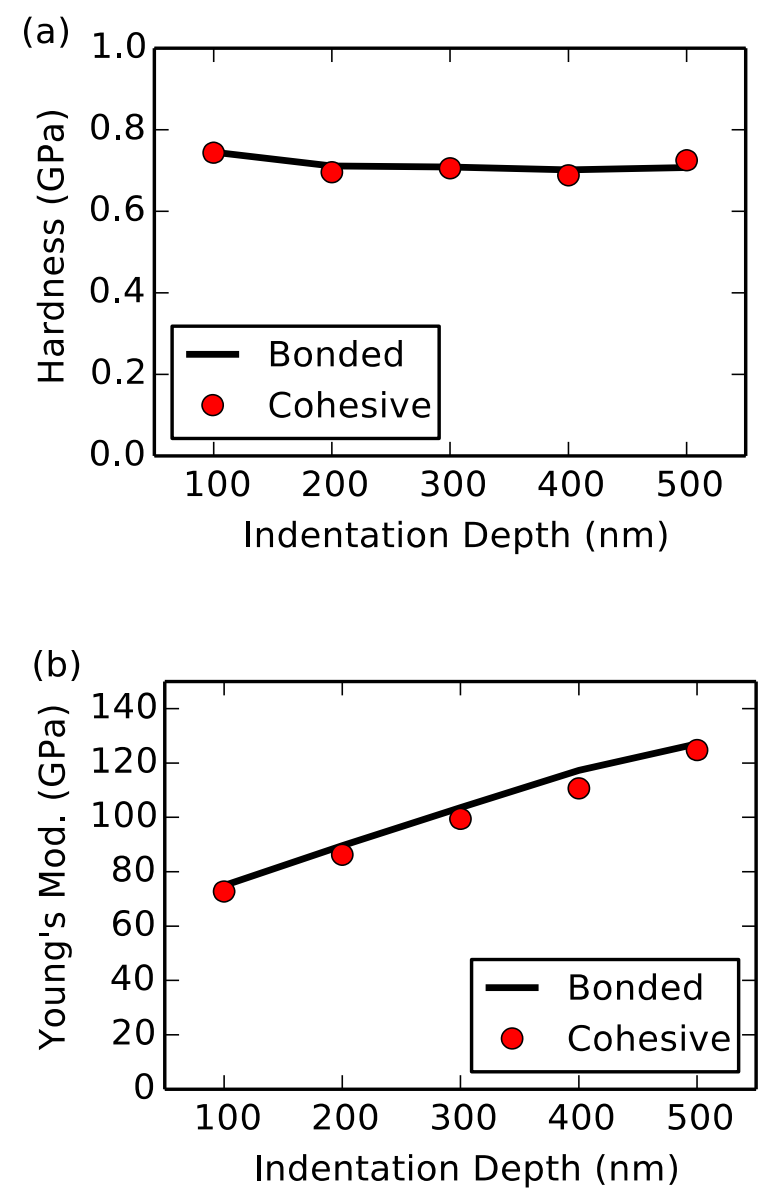

Fig. 5 Indentation-derived (a) hardness and (b) Young's modulus of a single-layer Al film with and without cohesive elements. "Bonded" refers to a perfect interface (no cohesive elements) and "Cohesive" refers to an interface composed of cohesive elements. 
(a)

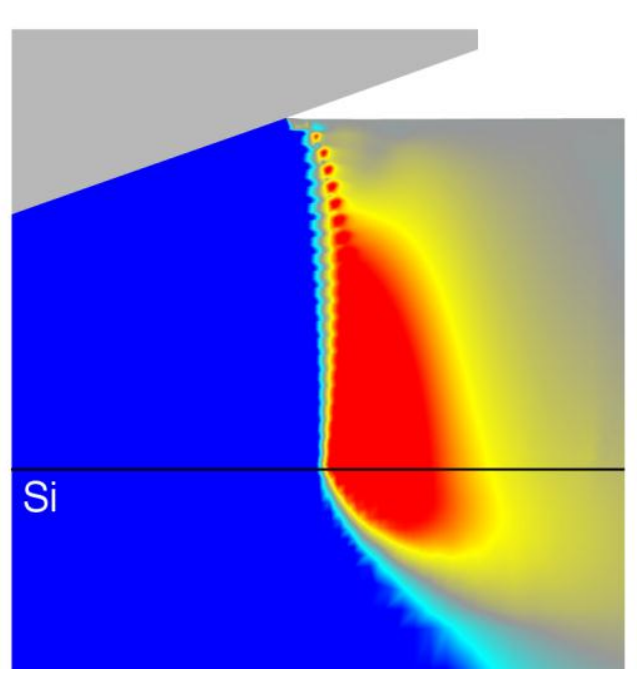

(b)

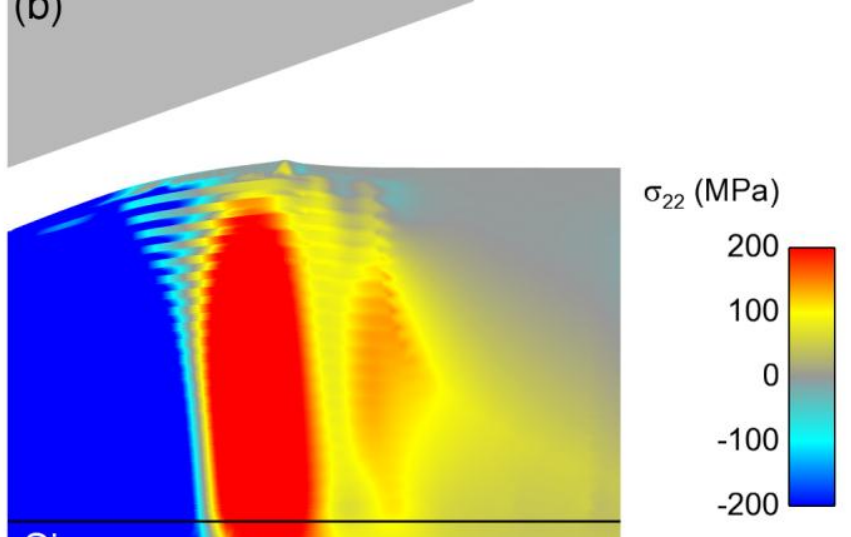

Fig. 6 Contour plots of axial stress $\sigma_{22}$ (along the indentation direction) in the perfectly bonded multilayer $\mathrm{Al} / \mathrm{SiC}$ model, when (a) the indentation reaches its peak depth at $600 \mathrm{~nm}$, and (b) after complete unloading. 
(a)

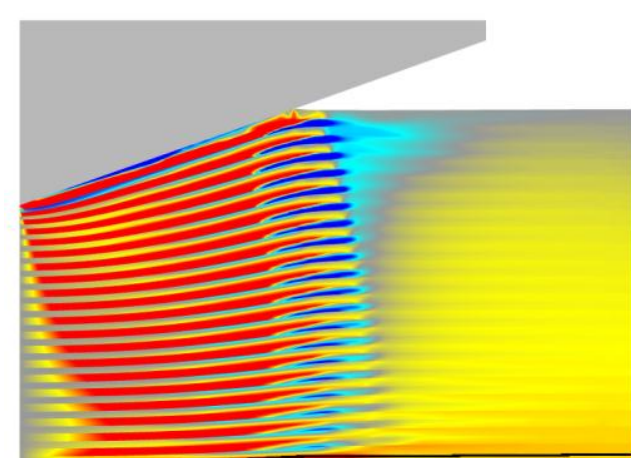

(b)
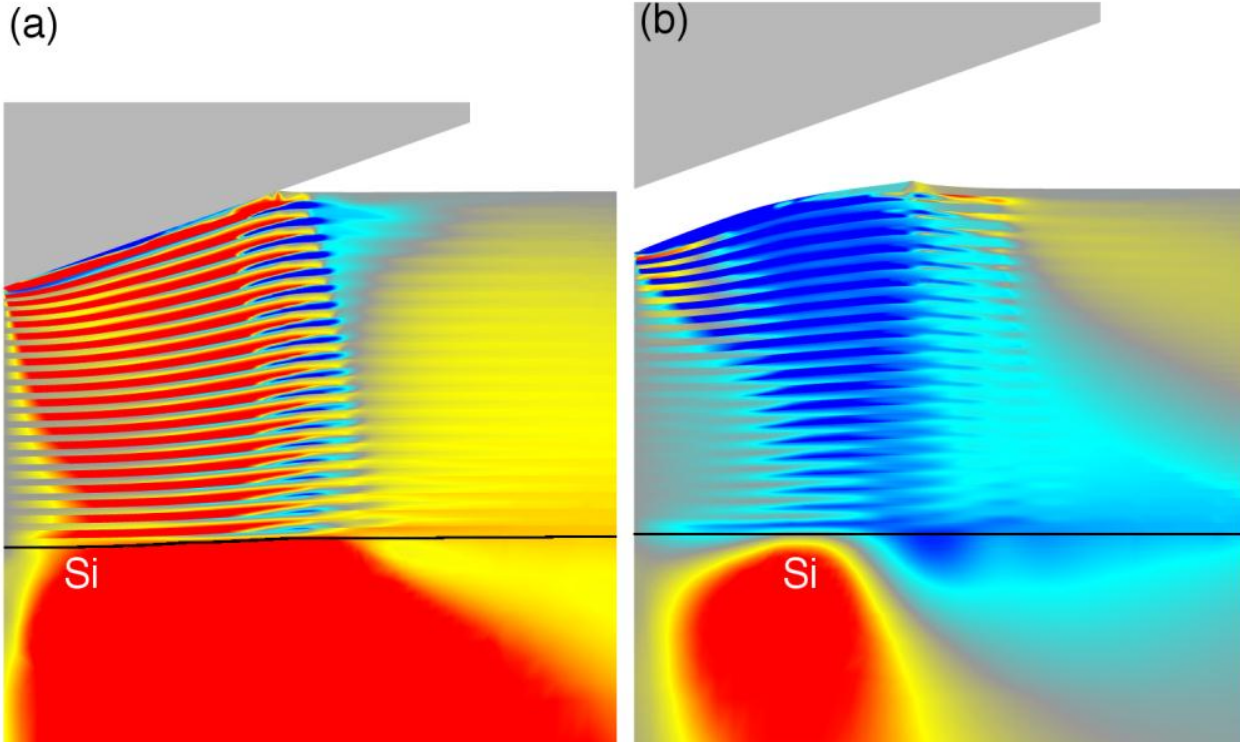

$\sigma_{12}(\mathrm{MPa})$

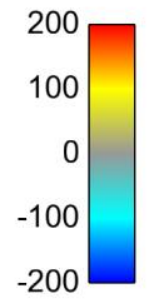

Fig. 7 Contour plots of shear stress $\sigma_{12}$ in the perfectly bonded multilayer $\mathrm{Al} / \mathrm{SiC}$ model, when (a) the indentation reaches its peak depth at $600 \mathrm{~nm}$, and (b) after complete unloading. 

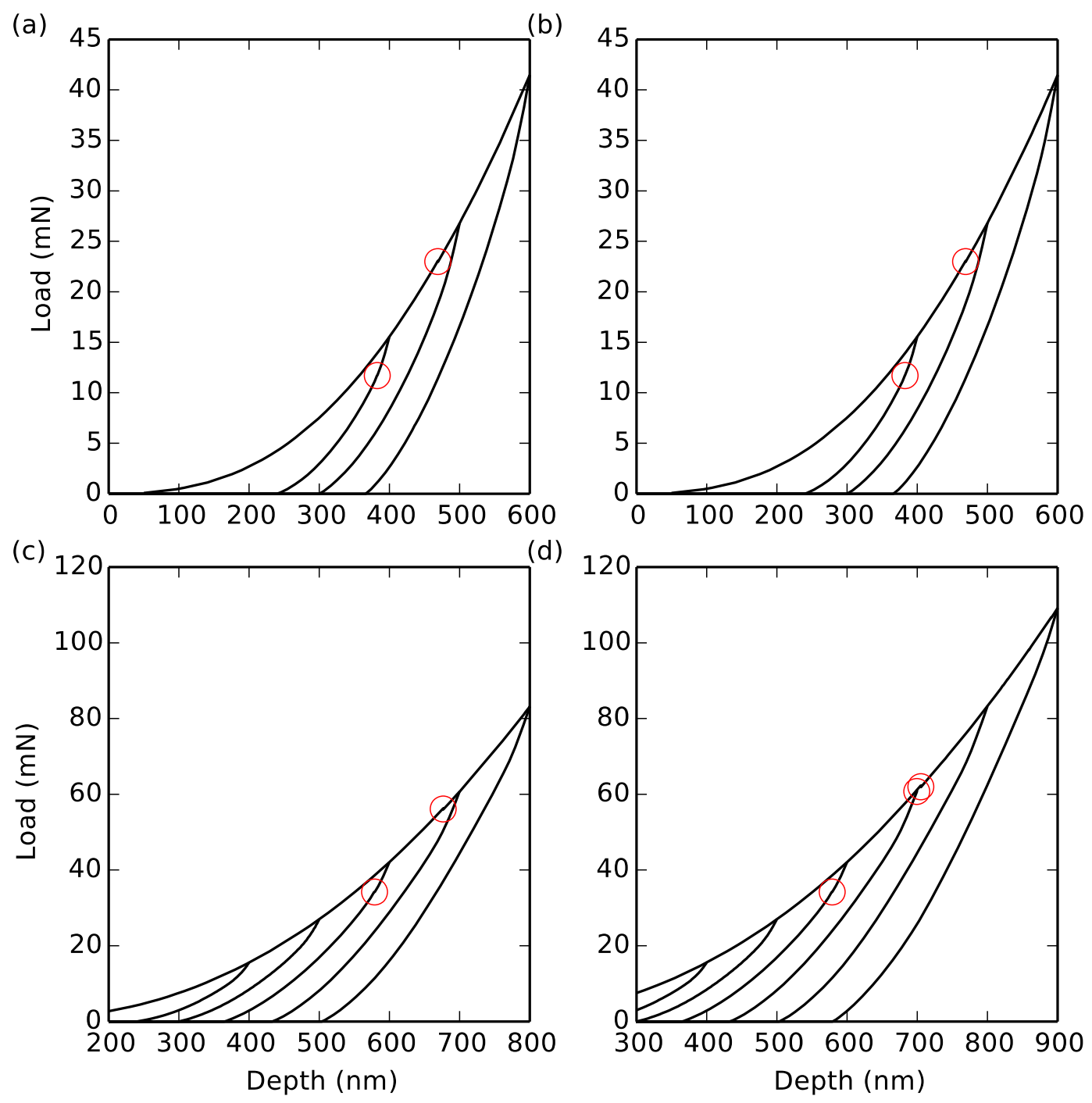

Fig. 8 The indentation load-displacement response of the multilayer $\mathrm{Al} / \mathrm{SiC}$ coating with cohesive elements at the $\mathrm{Al} / \mathrm{Si}$ interface with variations in normal and shear critical tractions. (a) $t_{n}^{0}=200 \mathrm{MPa}, t_{s}^{0}=200 \mathrm{MPa}$ (b) $t_{n}^{0}=200 \mathrm{MPa}, t_{s}^{0}=400 \mathrm{MPa}$ (c) $t_{n}^{0}=400$ $\mathrm{MPa}, t_{s}^{0}=200 \mathrm{MPa}$ (d) $t_{n}^{0}=400 \mathrm{MPa}, t_{s}^{0}=400 \mathrm{MPa}$. Circles denote the load-depth instances at which delamination occurs. 


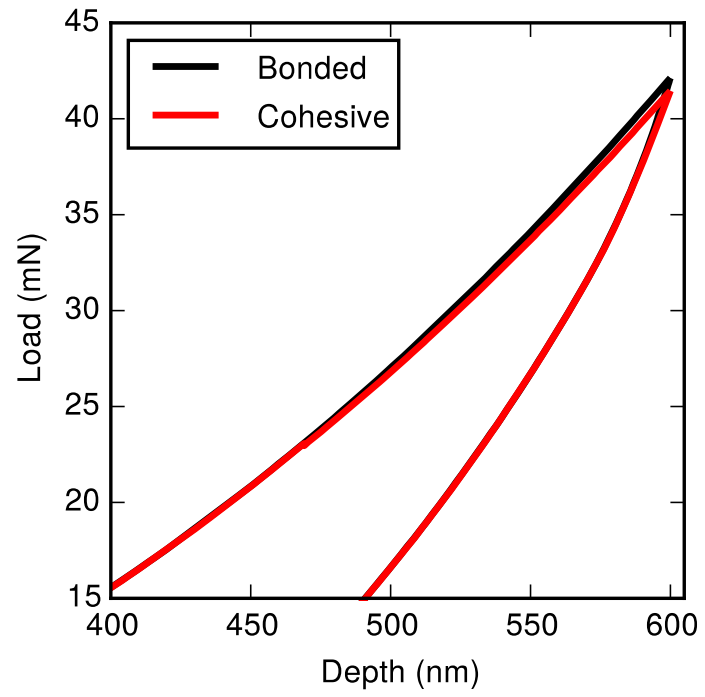

Fig. 9 Indentation load-displacement response of the multilayer $\mathrm{Al} / \mathrm{SiC}$ coating with cohesive elements at the $\mathrm{Al} / \mathrm{Si}$ interface, with a maximum indentation depth of $600 \mathrm{~nm}$. 


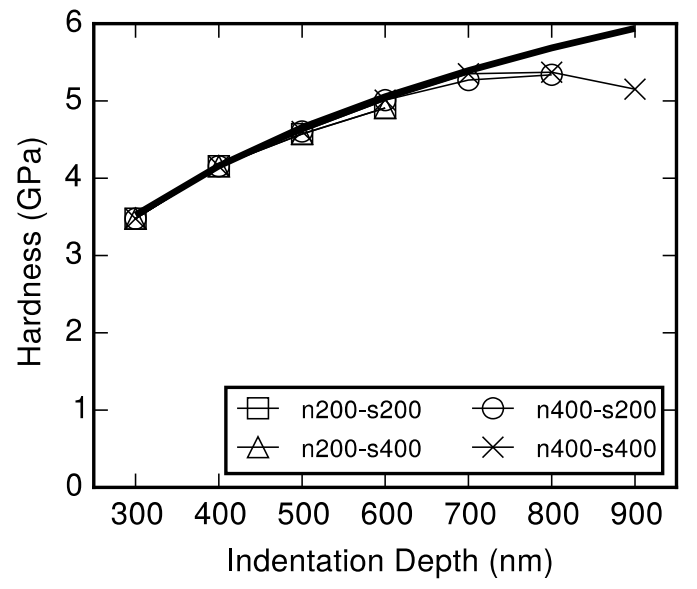

Fig. 10 Indentation-derived hardness of the multilayer $\mathrm{Al} / \mathrm{SiC}$ coating for a range of critical normal and shear tractions. The labels "n200-s200", "n200-s400", "n400-s200", and "n400-s400" represent the critical normal (n) and shear (s) tractions at the coating/substrate interface. The perfectly bonded model is the solid line. 


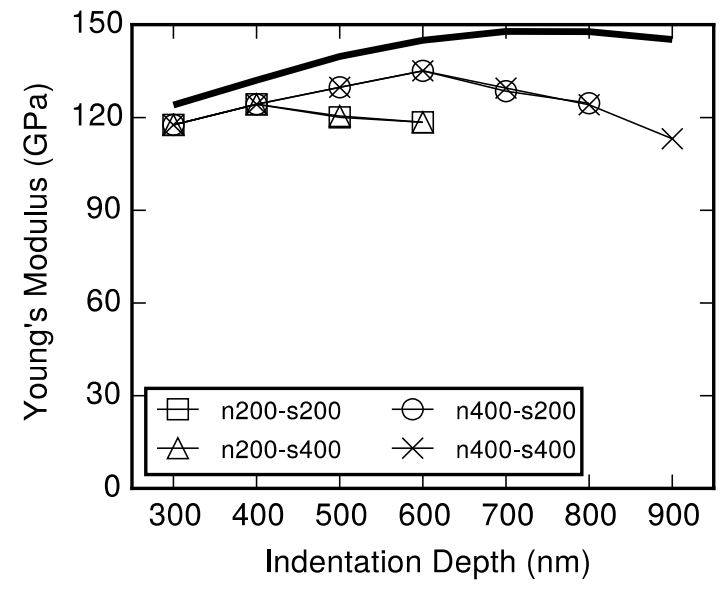

Fig. 11 Indentation-derived Young's modulus of the multilayer $\mathrm{Al} / \mathrm{SiC}$ coating for a range of critical normal and shear tractions. The labels "n200-s200", "n200-s400", "n400-s200", and "n400-s400" represent the critical normal (n) and shear (s) tractions at the coating/substrate interface. The perfectly bonded model is the solid line. 

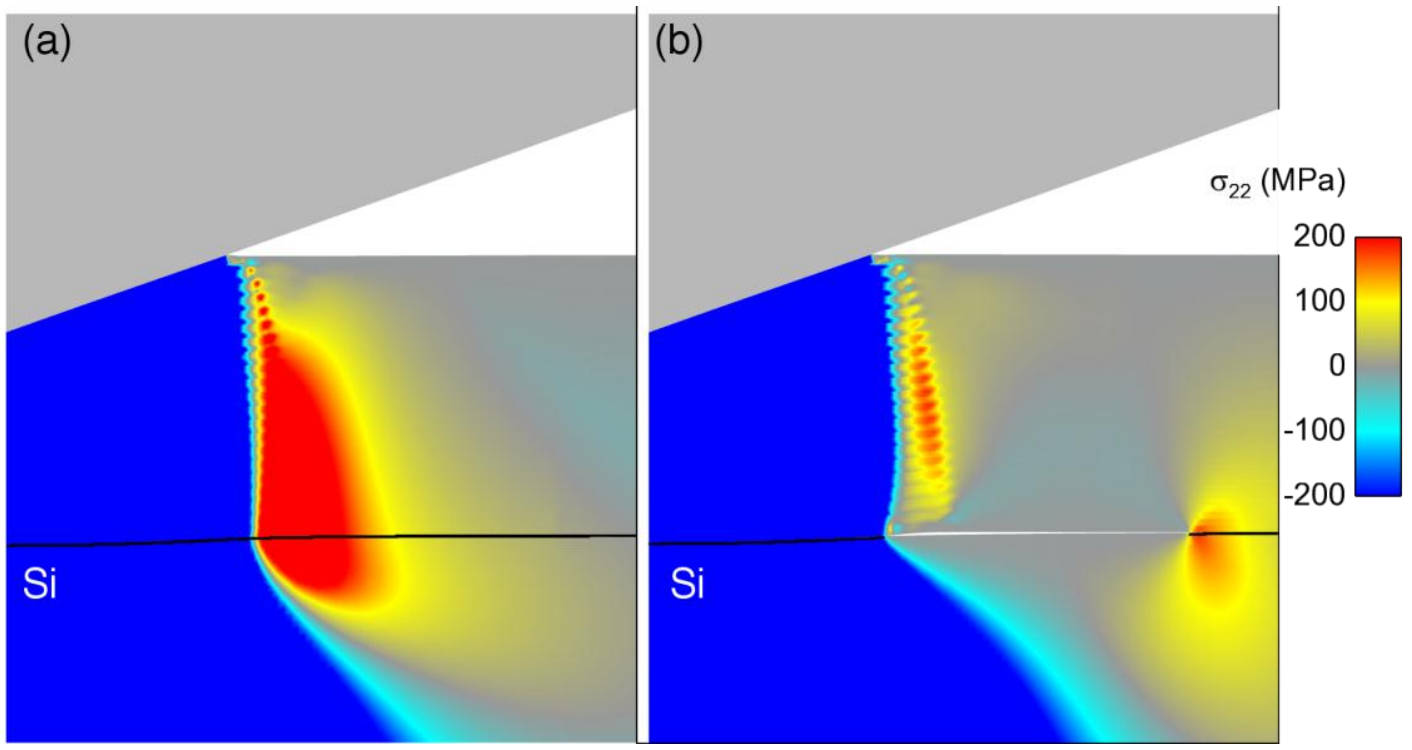

Fig. 12 Contour plots of axial stress $\left(\sigma_{22}\right)$ at $98 \%$ of the peak load during unloading, for a peak indentation depth of $600 \mathrm{~nm}$ in the models with (a) perfect bonding and (b) cohesive elements at the coating/substrate interface. 


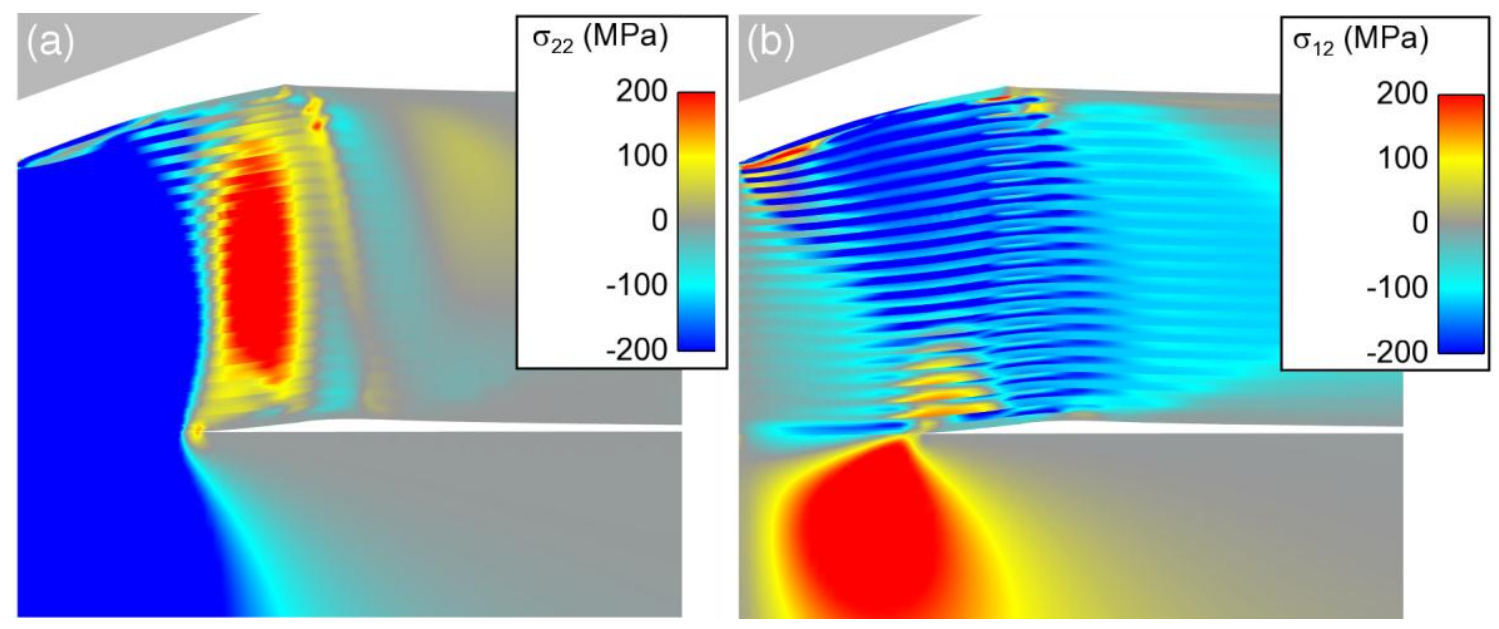

Fig. 13 Contour plots of (a) axial stress $\sigma_{22}$ and (b) shear stress $\sigma_{12}$ in the multilayer $\mathrm{Al} / \mathrm{SiC}$ structure after complete unloading from the maximum indentation depth of $600 \mathrm{~nm}$. Unloading is seen to enhance the opening at the coating/substrate interface. 

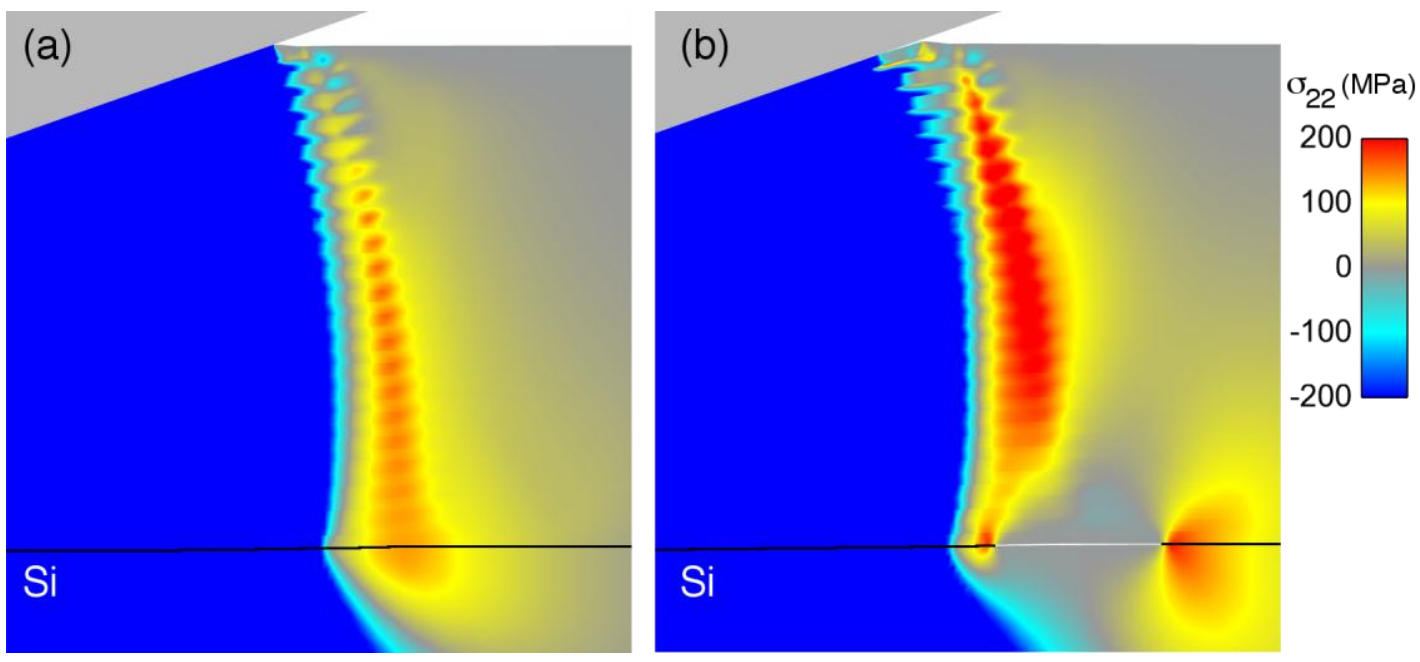

Fig. 14 Contour plots of axial stress $\sigma_{22}$ in the multilayer $\mathrm{Al} / \mathrm{SiC}$ structure (a) at the peak indentation depth of $400 \mathrm{~nm}$ and (b) after unloading to $10.9 \mathrm{mN}$. Delamination in the case is completely generated during unloading. 